\title{
WHEN SIMULATION BECOMES HUMAN CENTRIC ANALYTICS
}

\author{
Dr Christina J. Phillips \\ Liverpool John Moores University \\ Liverpool Business School, Redmonds Building, Brownlow Hill, Liverpool L3 5UG \\ c.j.phillips@1jmu.ac.uk
}

\begin{abstract}
In the following we describe a recent development in the integration of analytics into human processes called Human Centric Analytics (HCA), and take a look at how simulation fits into the design process that lies at the heart of HCA. The article will outline what HCA is, and how it works, and how simulation can facilitate its development. Referring to literature from OR and Design we show how simulation can become a mutually designed controller, forming part of a cybernetic evolutionary process toward improved ends and increased knowledge. Simulation is capable of facilitating more than an end artefact for experimental design and analyses, as it can provide a focus for group agency and an opportunity to see into processes and problems in novel and creative ways.
\end{abstract}

Keywords: Analytics, Human Centred Design, Participation, Cybernetics

\section{INTRODUCTION}

Analytics is described as a dianoetic management system that enables management decisions via a fusion of technology, quantitative methods and decision science (Mortenson, Doherty and Robinson, 2015). On the analytics stack of descriptive, predictive and prescriptive methods (Davenport and Harris, 2007) simulation is used mainly in predictive tasks but can be useful across the stack. In the era of big data and digital twins, simulation has become an important part of a firms analytics capabilities but there are still many challenges to overcome (Fowler and Rose, 2004).

In the realm of healthcare, participative simulation has been used effectively to solve problems, implement lean, create knowledge and increase awareness (Robinson et al., 2012; Kotiadis, Tako and Vasilakis, 2013; Pessôa et al., 2015; Baril et al., 2016; Lamé, Jouini and Stal-Le Cardinal, 2019), to a lesser extent the same thing has been observed in industry (Holweg and Bicheno, 2002; Abdulmalek and Rajgopal, 2007; Pool, Wijngaard and Van der Zee, 2011; Phillips and Nikolopoulos, 2019). Many researchers have noted that the process of performing the participative simulation can be as informative as the end artefact (Robinson, 2001; Holweg and Bicheno, 2002; Pool, Wijngaard and Van der Zee, 2011). This is where simulation becomes human centric and cybernetic as knowledge is created in the moment across a surface of becoming (Pickering, 2009). The process then becomes as important as the end product, and this means we need tools with which to study and perform it well (Mingers and Brocklesby, 1997; Ormerod, 1997; Yearworth and White, 2014).

\section{HUMAN CENTRIC ANALYTICS}

HCA is a design paradigm for analytics that involves the human users and producers of data within the design process as completely as possible (Phillips, 2019). It requires a cross disciplinary stance, which mixes methodologies and positivist and interpretive paradigms, to create a technique that bridges the technical and social domains. It is not proscriptive in the methods used since it is a paradigmatic stance that requires high human involvement, iterations of design, effective communication, and an understanding of context and multiple viewpoints. The simplicity of the idea belies the complexity of working with human actors, technologies and systems to create analytical artefacts, including appropriate data pipelines. The technique necessarily mixes methodologies and in this respect needs to maintain, what Lane and Oliva (1994) call 'dynamic coherence' as stakeholders are alternately exposed 


\section{Phillips}

to suggested changes that instigate instinctive reactions, or behaviour induced by possible causal structure, via the modelling process.

\section{THE CYBERNETIC SYSTEM}

The cybernetic view of design as modelling is explored by Maier et al. (2014) and Maier et al. (2012), viewing designing as a cybernetic system regulated by methods and process models. Cybernetics is taken from the Greek Kybernetes and means helmsman or steersman (Ashby, 1956). It is a paradigm that views systems as self-regulating via controllers, iterations and information flows. It has an almost hylozoist view of human-machine systems, ignoring boundaries and viewing the socio-technical in a holistic way. Studies in cybernetics have shown how one can get exceedingly complex and unpredictable behaviour from iterative interactions of relatively simple inputs (Beer, 1966; Pickering, 2009).

As simulators we know that to try to model every detail is, in general, both futile and unnecessary (Robinson, 2014). Frequently clients do not recognise this, and there is also a modelling process of problem simplification which to us is intuitive but which can provide unexpected insight for stakeholders into previously messy and intractable problem domains (Phillips and Nikolopoulos, 2019). Simulation models and the accompanying analyses that help to determine data and parameters provide a means of group derived agency which can communicate with others, be that the modeller/designer, the system itself or different functions and hierarchies within an organisation (Eckert, Maier and McMahon, 2005). From a cybernetic viewpoint when we model in a participative and iterative way we are adapting to create a future collaboratively at each step. The models have to be simple to achieve a general applicability, to achieve a complete system model we would have to copy the requisite variety of the system (Ashby, 1956) which would be overly detailed and too specific (Maier et al., 2012) to be useful.

Providing views, which work to gain insight, requires methods and models that are not overly prescriptive so that they are open to multiple interpretation, both as a group and individually, and the ambiguity of situations with high human involvement, and multiple viewpoints, requires simple and parsimonious simulation models (Robinson et al., 2014). As a cybernetic system, simulation modelling needs the ability to be reactive and reflexive in the face of change that comes about as part of the design process. HCA must be reflexive and dynamic or it cannot incorporate the creativity of the stakeholders as part of the design, since they are the subject matter experts it is their creativity and free moves in overcoming resistance to change that enable analytics which augment their work (Pickering, 1995; Phillips, 2019a). These moments are the bridging of existing human practice with novel technologies and data views, which in turn facilitate an evolution of knowledge and design. The design process brings about a certain level of unpredictability, which in a very cybernetic way should be embraced and accepted (Pickering, 2009).

\section{MIXING METHODS}

PartiSim (Tako and Kotiadis, 2015) fosters HCA as it uses the broad bounded, yet complete methodology of SSM combined with DES. Due to its multiple iterations of design with high human involvement, in as much of the process as possible, it is a natural vehicle for HCA. Phillips and Nikolopoulos (2019), used PartiSim in a manufacturing environment which had unexpected consequences due to the simple contextualised models which were needed to facilitate participation throughout. They provided visualisations and simple analysis to allow stakeholders to choose model parameters in an informed and inclusive way. This prompted improvements in forecasting as well as to the planning and scheduling process under study. Their end model was used by senior management to make major strategic decisions regarding the factory, something which has been lacking in OR applications (Lane, 2010), and the forecasting improvements helped the company make large inventory savings. In this case the participative simulation development had multiple consequences and these were enabled by the simple yet well-structured constitutive rules of PartiSim, which were robust in the face of change and easily flexed as necessary.

SSM has been used by many simulation modellers and others in mixed methods research that bridges the social and the technical (Lane and Oliva, 1994; Tako and Kotiadis, 2015; Small and 
Wainwright, 2018; Lamé, Jouini and Stal-Le Cardinal, 2019). The SSM methodologies have a strong underlying philosophy and lend themselves to being decomposed into separate parts, which can be cherry picked for situational suitability (Mingers and Brocklesby, 1997). This also allows the methods to be kept simple yet flexible as the cybernetic view would suggest they need to be. Lamé, Jouini and Stal-Le Cardinal (2019) combine DES and SSM, but also found that ethnography provided a way to both study the system under consideration and to provide an objective perspective to the stakeholders involved in the study. They used Analyses I, II, and III, and root definitions from SSM (Checkland, 1999), Phillips (2019b) had root definitions predefined and found the CATWOE (Customers, Actors, Transformation process, Worldviews, Owners, Environment) to be most useful. Pessôa et al. (2015) mixed methods using cognitive mapping and DES to involve experts in simulation experiments, leading to problem solution and improvement. They did not use any of the rest of the SODA method usually associated with cognitive mapping (Eden, 1988).

\section{CONCLUSION}

When simulation modellers handle the social and political elements of participative simulation exercises, via mixed methodologies, there are often insights that go beyond the initial intention of the exercise. The simulation activity becomes a performative process and the models become facilitators of thought, knowledge creation, and creativity, encouraging ideation in a group setting. Until the end simulation is settled upon there is a continuous design process. This can foster lean and continuous improvement (Holweg and Bicheno, 2002; Abdulmalek and Rajgopal, 2007; Van der Zee, Pool and Wijngaard, 2008; Pool, Wijngaard and Van der Zee, 2011; Phillips and Nikolopoulos, 2019) by providing a focus of ideation, and a possibility to create an artefact derived via group agency.

Phillips (2019), used PartiSim to improve testing and scheduling in a pharmaceutical factory, but also used action research (Eden and Ackermann, 2018) and intervention theory (Argyris, 1970), to take the simulation from working with shop floor stakeholders to providing a decision tool for senior managers. These additional intervention techniques maintained a research framework to provide structural and ethical/social/political guidance as well as recoverable information from the many human centric interactions.

Techniques such as ethnomethodology (Franco and Greiffenhagen, 2018), ethnography (Lamé, Jouini and Stal-Le Cardinal, 2019), symbolic interaction theory (Gallant and Kleinman, 1983) and the mangle of practice (Pickering, 1995; Ormerod, 2014) can help us to see into the practice of simulation with high human involvement, moving forward theory around participative simulation and HCA.

Perhaps one way to overcome Fowler and Rose's (2004) last and most difficult 'grand challenge'; that of persuading managers to engage more with simulation, is to help them see it as a vital part of the analytics stack. In particular, as a means to foster HCA that can not only increase knowledge and provide useful artefacts, but that can also shift a culture toward being more proactive and data curious (Phillips, 2019).

\section{REFERENCES}

Abdulmalek, F. A. and Rajgopal, J. (2007) 'Analyzing the benefits of lean manufacturing and value stream mapping via simulation: A process sector case study', International Journal of Production Economics, 107(1), pp. 223-236. doi: 10.1016/j.ijpe.2006.09.009.

Baril, C. et al. (2016) 'Use of a discrete-event simulation in a Kaizen event: A case study in healthcare', European Journal of Operational Research. Elsevier Ltd., 249(1), pp. 327-339. doi: 10.1016/j.ejor.2015.08.036.

Davenport, T. H. and Harris, J. G. (2007) Competing on Analytics: The New Science of Winning. 1st edn. Boston, MA: Harvard Business School Press. Available at: http://dl.acm.org/citation.cfm?id=1526366 (Accessed: 13 March 2017).

Fowler, J. W. and Rose, O. (2004) 'Grand Challenges in Modeling and Simulation of Complex Manufacturing Systems', Simulation, 80(9), pp. 469-476. doi: 10.1177/0037549704044324.

Franco, L. A. and Greiffenhagen, C. (2018) 'Making OR practice visible: Using ethnomethodology to analyse facilitated modelling workshops', European Journal of Operational Research. NorthHolland, 265(2), pp. 673-684. doi: 10.1016/J.EJOR.2017.08.016. 


\section{Phillips}

Gallant, M. J. and Kleinman, S. (1983) 'Symbolic Interactionism vs. Ethnomethodology', Symbolic Interaction, 6(1), pp. 1-18.

Holweg, M. and Bicheno, J. (2002) 'Supply chain simulation - A tool for education, enhancement and endeavour', International Journal of Production Economics, 78(2), pp. 163-175. doi: 10.1016/S0925-5273(00)00171-7.

Kotiadis, K., Tako, A. A. and Vasilakis, C. (2013) 'A participative and facilitative conceptual modelling framework for discrete event simulation studies in healthcare', Journal of the Operational Research Society, 65(2), pp. 197-213. doi: 10.1057/jors.2012.176.

Lamé, G., Jouini, O. and Stal-Le Cardinal, J. (2019) 'Combining Soft Systems Methodology, ethnographic observation, and discrete-event simulation: A case study in cancer care', Journal of the Operational Research Society. Taylor \& Francis, 0(0), pp. 1-18. doi: 10.1080/01605682.2019.1610339.

Lane, D. C. and Oliva, R. (1994) 'The Greater Whole: Towards a synthesis of SD and SSM', 1994 International System Dynamics Conference, p. 134.

Mingers, J. and Brocklesby, J. (1997) 'Multimethodology: Towards a framework for mixing methodologies', Omega, 25(5), pp. 489-509. doi: 10.1016/S0305-0483(97)00018-2.

Mortenson, M. J., Doherty, N. F. and Robinson, S. (2015) 'Operational research from Taylorism to Terabytes: A research agenda for the analytics age', European Journal of Operational Research. Elsevier Ltd., 241(3), pp. 583-595. doi: 10.1016/j.ejor.2014.08.029.

Ormerod, R. J. (1997) 'The role of OR in shaping the future: smart bits, helpful ways and things that matter', Journal of the Operational Research Society, 48(11), pp. 1045-1056. doi: 10.1057/palgrave.jors.2600465.

Pessôa, L. A. M. et al. (2015) 'Integrating soft and hard operational research to improve surgical centre management at a university hospital', European Journal of Operational Research, 245(3), pp. 851861. doi: 10.1016/j.ejor.2015.04.007.

Phillips, C. J. (2019) Human Centric Analytics: Creating a data driven culture to manage uncertainty (under revision EJOR).

Phillips, C. J. and Nikolopoulos, K. (2019) 'Forecast quality improvement with Action Research: A success story at PharmaCo', International Journal of Forecasting. Elsevier B.V., 35(1), pp. 129143. doi: 10.1016/j.ijforecast.2018.02.005.

Pickering, A. (2009) 'Beyond design: Cybernetics, biological computers and hylozoism', Synthese, 168(3), pp. 469-491. doi: 10.1007/s11229-008-9446-z.

Pool, A., Wijngaard, J. and Van der Zee, D.-J. (2011) 'Lean planning in the semi-process industry, a case study', International Journal of Production Economics. Elsevier, 131(1), pp. 194-203. doi: 10.1016/j.ijpe.2010.04.040.

Robinson, S. (2001) 'Soft with a hard centre: Discrete-event simulation in facilitation', Journal of the Operational Research Society, 52(8), pp. 905-915. doi: 10.1057/palgrave.jors.2601158.

Robinson, S. et al. (2012) 'SimLean: Utilising simulation in the implementation of lean in healthcare', European Journal of Operational Research. Elsevier B.V., 219(1), pp. 188-197. doi: 10.1016/j.ejor.2011.12.029.

Yearworth, M. and White, L. (2014) 'The non-codified use of problem structuring methods and the need for a generic constitutive definition', European Journal of Operational Research. Elsevier B.V., 237(3), pp. 932-945. doi: 10.1016/j.ejor.2014.02.015.

\section{AUTHOR BIOGRAPHY}

CHRISTINA J. PHILLIPS is a Senior Lecturer in Business Analytics at Liverpool Business School and a Visiting Researcher at both Leeds and Bangor Universities. She gained the Siemens \& CBLESS studentship for an industrial research placement which gave her first-hand experience of participative modelling in a real world setting. Her previous experience, first as a manager, then as a designer and artist came into use more than expected, culminating in observations on the design paradigm of Human Centric Analytics. https://www.linkedin.com/in/christinajphillips/ 